\title{
RÍO BRAVO C2018, NUEEVA VARIEDAD DE TRIGO MACARRONERO PARA ÁREAS DE RIEGO EN MÉXICO
}

\author{
RÍO BRAVO C2018, A NEW VARIETY OF DURUM WHEAT \\ FOR IRRIGATED AREAS IN MEXICO
}

\begin{abstract}
Héctor E. Villaseñor-Mir ${ }^{1}$, Julio Huerta-Espino ${ }^{1}$, Eliel Martínez-Cruz ${ }^{1} *$, René HortelanoSanta Rosa', María F. Rodríguez-García', Ernesto Solís-Moya², Jorge I. AlvaradoPadilla $^{3}$, Alberto Borbón-Gracia ${ }^{4}$, Gabriela Chávez-Villalba ${ }^{5}$, Héctor Cortinas-Escobar ${ }^{6}$, Eduardo Espitia-Rangel' ${ }^{1}$ Leodegario Osario-Alcalá ${ }^{7}$ y Juan Martínez-Medina ${ }^{8}$
\end{abstract}

\begin{abstract}
IInstituto Nacional de Investigaciones Forestales, Agrícolas y Pecuarias (INIFAP), Campo Experimental Valle de México, Coatlinchán, Texcoco, Estado de México, México. ${ }^{2}$ NIFAP, Campo Experimental Bajío, Celaya, Guanajuato, México. ${ }^{3}$ INIFAP, Campo Experimental Valle de Mexicali, Mexicali, Baja California, México. ${ }^{4}$ INIFAP, Campo Experimental Valle del Fuerte, Guasave, Sinaloa, México. ${ }^{5}$ INIFAP, Campo Experimental Norman E. Borlaug, Cd. Obregón, Sonora, México. ${ }^{6}$ INIFAP, Campo Experimental Río Bravo, Tamaulipas, México. ${ }^{7}$ INIFAP, Campo Experimental Valles Centrales de Oaxaca, Santo Domingo Barrio Bajo, Etla, Oaxaca, México. ${ }^{8}$ INIFAP, Campo Experimental General Terán, General Terán, Nuevo León, México.

*Autor de correspondencia (martinez.eliel@inifap.gob.mx)
\end{abstract}

La producción anual de trigo cristalino (Triticum durum Desf) en México es aproximadamente de 2 millones de t, de las cuales cerca de 700 mil se utilizan para consumo nacional y 1.3 millones de t se exportan (CANIMOLT, 2016). Los principales estados productores son Sonora, Sinaloa, Baja California, Guanajuato y Michoacán, que participan con 90 \% de la producción total (SIAP, 2020). En el Valle del Yaqui, que es la zona más importante de producción de trigo cristalino en el sur de Sonora, el rendimiento potencial promedio por hectárea es de 6.9 t con riego y en la fecha normal de siembra (Valenzuela-Antelo et al., 2018).

El $100 \%$ del cultivo de trigo cristalino en México se produce bajo condiciones de riego (SIAP, 2020). Los factores limitantes en la producción son la escasez de agua de riego (Salazar et al., 2012), así como la incidencia de royas (Puccinia spp.). El problema con enfermedades se agravó con la aparición de nuevas razas con mayor virulencia de roya amarilla (Puccinia striiformis f. sp. tritici Eriks) y de roya de la hoja (Puccinia triticina), las cuales fueron reportadas en 2014 y 2017, respectivamente. Estas enfermedades vencieron la resistencia de la mayoría de las variedades sembradas en México (Pérez-López et al., 2017; Solís et al., 2016), lo que ha requerido la aplicación de fungicidas para su control.

Por otro lado, los industriales nacionales e internacionales demandan grano cristalino con mayor cantidad de pigmento amarillo y de proteína (ChávezVillalba et al., 2018). Una opción para hacer frente a la problemática de la producción del trigo cristalino, que engloba factores bióticos, abióticos, así como la demanda de los productores y de la industria de pastas alimenticias, es la liberación de nuevas variedades que conjunten alto rendimiento, tolerancia a las royas y buena calidad para la elaboración de pastas alimenticias (Magallanes-López et al., 2017). Bajo este contexto, el programa de trigo del Instituto Nacional de Investigaciones Forestales, Agrícolas y Pecuarias (INIFAP) pone a disposición de los productores de trigo de riego en México la variedad Río Bravo C2018, que es de alto rendimiento, resistente a las razas de roya prevalecientes en México y de buena calidad industrial.

La caracterización fenotípica de la nueva variedad se realizó de acuerdo con los descriptores de la Unión Internacional para la Protección de las Obtenciones Vegetales (UPOV). La descripción se realizó durante los ciclos de otoño-invierno (O-I) en Roque, Guanajuato y de primavera-verano $(P-V)$ en Chapingo, Estado de México. El INIFAP recibió el número de registro definitivo TRI-158290916 en el Catálogo Nacional de Variedades Vegetales del Servicio Nacional de Inspección y Certificación de Semillas y el titulo de obtentor 2312 para Rio Bravo C2018 en octubre de 2019.

Para obtener la línea, primero se realizó una cruza simple entre los progenitores parental A: TRN//D21563/AA/3/ BD2080/4/BD2339/5/RASCON37*2/TARR02/6/AUK/ GUIL//GREEN y parental B: ALTAR84ALTO1/RISSA/9/ USDA595/3/D67.3/RABI//CRA/4/ALO/5/HUI/YAV1/6/ ARDENTE/7/HUI/YAV79/8/POD9/10/SHAG14/ANADE1// KITTI1. La $F_{1}$ resultante se cruzó con la variedad Cirno C2008 (parental C). La cruza simple se realizó en el Batán, Texcoco, México en el ciclo P-V /2008, entre los progenitores identificados como parental A y parental B. La $F_{1}$ obtenida se sembró en Cd. Obregón, Sonora, México en el ciclo 0-I /2008-09 y se cruzó con la variedad Cirno C2008. La semilla de la cruza trilineal se identificó con el 
número CDSS09Y00939T, se sembró en la generación $F_{1}$ Top en el Batán, Texcoco en el ciclo P-V/2009 y se cosechó masivamente. La generación $F_{2}$ fue sembrada en Cd. Obregón en el ciclo 0-I/2009-10, se seleccionaron plantas individuales y se cosecharon en masa (099Y). La generación $\mathrm{F}_{3}$ fue sembrada en Toluca, Edo. de México en el ciclo P-V/2010, se seleccionaron 12 plantas y se trillaron en masa (012M). La generación $\mathrm{F}_{4}$ fue sembrada en Cd. Obregón en el ciclo O-I/2010-11, se seleccionaron plantas y se identificó como $2 Y$. La generación $F_{5}$ fue sembrara en Toluca en el ciclo P-V/2011 y se cosechó en masa (OM). La $F_{6}$ fue sembrada en Cd. Obregón en el ciclo 0-1/2011-12, se seleccionaron cuatro espigas individuales, mismas que se trillaron en masa (04Y) para dar origen a la línea que se identificó con el siguiente pedigrí: CDSS09Y00939T-099Y-012M-2Y-0M-04Y.

Río Bravo C2018 presentó severidades máximas de 10 \% con reacción de resistencia para las diferentes royas. Así mismo, se probó en 80 experimentos en Sinaloa, Sonora, Baja California, Coahuila, Tamaulipas, Guanajuato, Michoacán y Oaxaca bajo condiciones de riego completo y riego limitado (se restringió el último riego de auxilio). Río Bravo C2018 superó de 8 a 15 \% el rendimiento promedio de todas las variedades testigo en ambas condiciones de humedad (Cuadro 1).

Río Bravo C2018 presentó valores promedio de peso de mil granos de $50.2 \mathrm{~g}$, peso hectolítrico de $82 \mathrm{~kg} \mathrm{hL}^{-1}$, contenido de proteína en grano cercano a 12 \%; así mismo, valores de color amarillo de la sémola mayores a 22 unidades (Figura 1), factor importante que favorece el color característico de las pastas. Dichas características se consideran de muy buena calidad y son adecuadas para la elaboración de pastas alimenticias.

La nueva variedad Río Bravo C2018 se recomienda para condiciones de riego y riego limitado durante el ciclo otoño-invierno. En los estados de Sonora, Sinaloa, Baja California, Chihuahua, Coahuila, Nuevo León y Tamaulipas se recomienda en fechas de siembras tempranas, durante los primeros 15 días de noviembre, a tardías, en la primera quincena de enero, mientras que para los estados de la zona de El Bajío se recomienda para siembras tempranas. La semilla de Río Bravo C2018 se encuentra disponible para su venta en el Campo Experimental Bajío del INIFAP.

\section{AGRADECIMIENTOS}

Al Programa de Mejoramiento de Trigos Macarroneros del CIMMYT por la generación de la línea, al FONSEC SAGARPA-CONACYT (Proyecto 146788) y a MASAGRO Proyecto WYC-2018-002 por el financiamiento de los ensayos nacionales y de la publicación.

\section{BIBLIOGRAFÍA}

CANIMOLT, Cámara Nacional de la Industria Molinera de Trigo (2016) Reporte Estadístico 2015 con Información al 2016. Cámara Nacional de la Industria Molinera de Trigo. Ciudad de México. $116 \mathrm{p}$.

Chávez-Villalba G., M. A. Camacho-Casas, K. Ammar, J. I. Alvaro-Padilla, G. Fuentes D. y A. Borbon-Gracia (2018) CENEB Oro C2017: nueva variedad de trigo duro para el noroeste de México. Revista Mexicana de Ciencias Agrícolas 9:560-1563, https://doi. org/10.29312/remexca.v9i7.1679

Magallanes-López A. M., K. Ammar, A. Morales-Dorantes, H. GonzálezSantoyo, J. Crossa and C. Guzmán (2017) Grain quality traits of commercial durum wheat varieties and their relationships with drought stress and glutenins composition. Journal of Cereal Science 75:1-9, https://doi.org/10.1016/j.jcs.2017.03.005

Pérez-López J. B., E. García-León, H. E. Villaseñor-Mir, P. R. Singh y K. Ammar (2017) Desarrollo de la epifitia de roya de la hoja causada por Puccinia triricina E. en la variedad de trigo cristalino Cirno C2008 durante el ciclo otoño-invierno 2016-2017 en el Bajo Río Mayo, Sonora. Revista Mexicana de Fitopatología 35 (Supl. 2017):S39.

Salazar A. A., J. L. Moreno V. y A. N Lutz L. (2012) Agricultura y manejo sustentable del acuífero de la Costa de Hermosillo. Región y Sociedad 24:155-179, https://doi.org/10.22198/ rys.2012.3.a411

Cuadro 1. Promedio de rendimiento de grano y reacción a royas de Río Bravo C2018 y variedades testigo bajo condiciones de riego completo y riego limitado evaluadas en 80 experimentos durante los ciclos de 0-1/2013-2014 a 0-1/2017-2018.

\begin{tabular}{lccccc}
\hline \multirow{2}{*}{ Variedad } & RRC & RRL & RH & RAH & RAE \\
\cline { 2 - 5 } & $\left(\mathrm{kg} \mathrm{ha}^{-1}\right)$ & $\left(\mathrm{kg} \mathrm{ha}^{-1}\right)$ & $(\% \mathrm{YS})$ & $(\% \mathrm{YS})$ & $(\%)$ \\
\hline Río Bravo C2018 & $6370 \mathrm{a}$ & $5163 \mathrm{a}$ & $0-10 \mathrm{R}$ & $0-10 \mathrm{R}$ & $0-5$ \\
Cevy oro C2008 & $5665 \mathrm{a}$ & $4748 \mathrm{a}$ & $0-30 \mathrm{MR}$ & $0-30 \mathrm{MS}$ & $0-30$ \\
Cemexi C2008 & $5387 \mathrm{a}$ & $4533 \mathrm{a}$ & $10 \mathrm{MR}-50 \mathrm{MS}$ & $0-20 \mathrm{MS}$ & $5-20$ \\
Anatoly C2011 & $5336 \mathrm{a}$ & $4756 \mathrm{a}$ & $0-50 \mathrm{MS}$ & $0-15 \mathrm{MR}$ & $0-5$ \\
Movas C2009 & $5418 \mathrm{a}$ & $4363 \mathrm{a}$ & $0-10 \mathrm{R}$ & $20 \mathrm{MR}-70 \mathrm{MS}$ & $0-40$ \\
DSH (0.05) & 999.1 & 1195.0 & & & \\
\hline
\end{tabular}

Medias con letras diferentes en la misma columna son estadísticamente diferentes (Tukey, $\mathrm{P} \leq 0.05$ ). RRC: rendimiento riego completo, RRL: rendimiento riego limitado, $\mathrm{RH}$ : roya de la hoja, $\mathrm{RAH}$ : roya amarilla en hoja, RAE: roya amarilla en espiga, R: resistente, MR: moderadamente resistente, MS: moderadamente susceptible, S: susceptible \% YS: porcentaje y calificación de severidad, DSH: diferencia significativa honesta. 


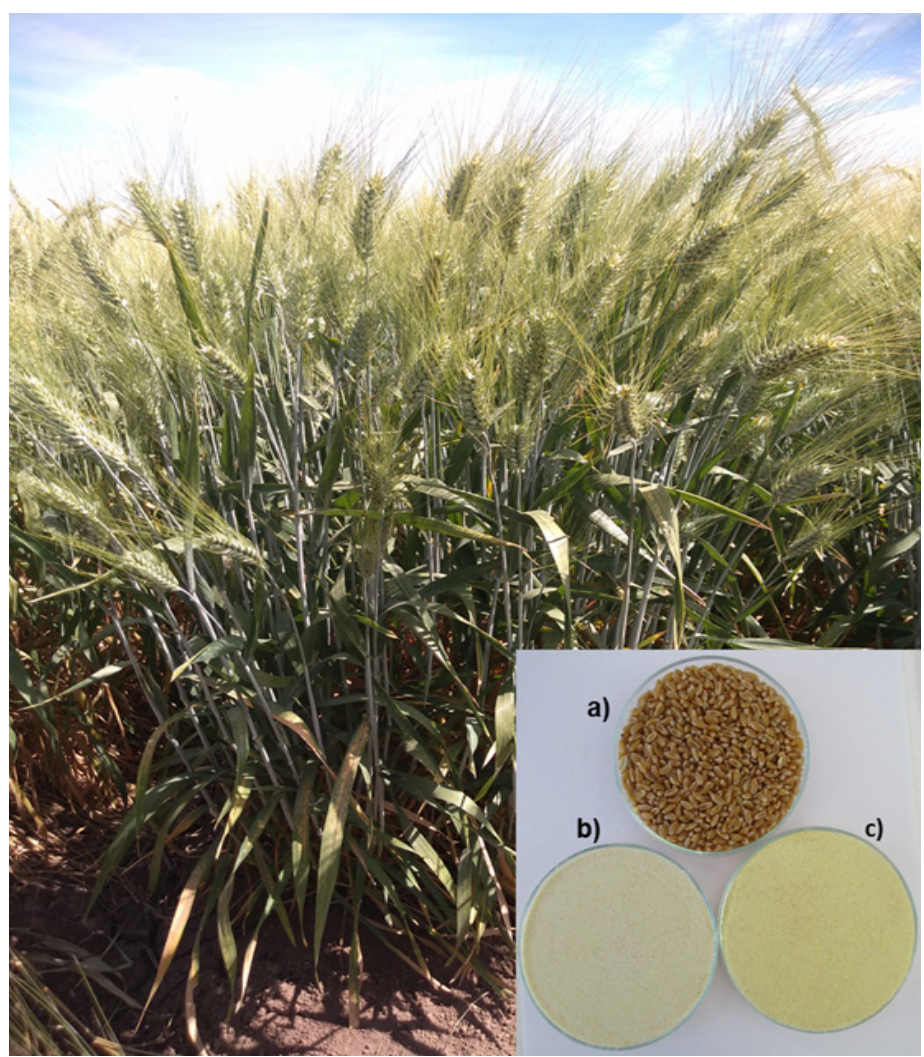

Figura 1. Aspecto de la planta y grano de Río Bravo C2018. a) Grano entero, b) sémola de calidad deficiente, c) sémola de calidad adecuada de Río Bravo C2018.

SIAP, Servicio de Información Agroalimentaria y Pesquera (2020) Boletín mensual de producción. Trigo. Servicio de Información Agroalimentaria y Pesquera. Secretaría de Agricultura y Desarrollo Rural. Ciudad de México. https://www.gob.mx/cms/ uploads/attachment/file/537083/Boletin_avance_producci_n_ trigo_enero_2020.pdf (Mayo 2020).

Solís M. E., J. Huerta E., P. Pérez H., H. E. Villaseñor M., A. Ramírez R. y M. L. De la Cruz G. (2016) Alondra F2014, nueva variedad de trigo harinero para el Bajío, México. Revista Mexicana de Ciencias Agrícolas 7:1225-1229, https://doi.org/10.29312/remexca.v7i5.248

Valenzuela-Antelo J. L., I. Benítez-Riquelme, H. E. Villaseñor-Mir, J. HuertaEspino, R. Lobato-Ortiz, G. Bueno-Aguilar y M. Vargas-Hernández (2018) Comparación del rendimiento de trigos harineros y cristalinos a través de diferentes ambientes de riego. Revista Fitotecnia Mexicana 41:159-166, https://doi.org/10.35196/ rfm.2018.2.159-166 
